\title{
miR-1293 acts as a tumor promotor in lung adenocarcinoma via targeting phosphoglucomutase 5
}

\author{
Bing Chen ${ }^{1,2}$, Shiya Zheng ${ }^{3}$, Feng Jiang ${ }^{\text {Corresp. } 1,2}$ \\ ${ }^{1}$ Department of Thoracic Surgery, Jiangsu Cancer Hospital \& Jiangsu Institute of Cancer Research, Nanjing, China \\ 2 Jiangsu Key Laboratory of Molecular and Translational Cancer Research, Cancer Institute of Jiangsu Province, Nanjing, China \\ 3 Department of Oncology, Zhongda Hospital, School of Medicine, Southeast University, Nanjing, China \\ Corresponding Author: Feng Jiang \\ Email address: fengjiang_nj@njmu.edu.cn
}

Background: Lung adenocarcinoma (LUAD) is the most common histologic subtype of lung cancer. Studies have found that miR-1293 is related to the survival of LUAD patients. Unfortunately, its role in LUAD remains not fully clarified. Methods: miR-1293 expression and its association with LUAD patients' clinical characteristics were analyzed in TCGA database. Also, miR-1293 expression was detected in LUAD cell lines. Cell viability, migration, invasion and expression of MMP2 and MMP9 were measured in LUAD cells following transfection with miR-1293 mimic or antagomir. Phosphoglucomutase (PGM) 5 was identified to be negatively related to miR-1293 in LUAD patients in TCGA database, and their association was predicated by Targetscan software. Hence, we further verified the relationship between miR-1293 and PGM5. Additionally, the effect and mechanism of miR-1293 were validated in a xenograft mouse model. Results: We found miR-1293 expression was elevated, but PGM5 was decreased, in LUAD patients and cells. Higher miR-1293 expression was positively related to LUAD patients' pathologic stage and poor overall survival. miR-1293 mimic significantly promoted, whereas miR-1293 antagomir suppressed the viability, migration, invasion, and expression of MMP2 and MMP9 in LUAD cells. PGM5 was a target of miR-1293. Overexpression of PGM5 abrogated the effects of miR-1293 on the malignant phenotypes of LUAD cells. Administration of miR-1293 antagomir reduced tumor volume and staining of Ki-67 and MMP9, but elevated PGM5 expression in vivo. Conclusions: miR-1293 promoted the proliferation, migration and invasion of LUAD cells via targeting PGM5, which indicated that miR-1293 might serve as a potential therapeutic target for LUAD patients. 
1 miR-1293 acts as a tumor promotor in lung

2 adenocarcinoma via targeting phosphoglucomutase 5

3

4

5

6

\section{7}

\section{Bing Chen ${ }^{1,2}$, Shiya Zheng ${ }^{3}$, Feng Jiang ${ }^{1,2}$}

1. The Affiliated Cancer Hospital of Nanjing Medical University, Nanjing, China \& Department of Thoracic Surgery, Jiangsu Cancer Hospital \& Jiangsu Institute of Cancer Research, Nanjing 210009, Jiangsu, China

2. Jiangsu Key Laboratory of Molecular and Translational Cancer Research, Cancer Institute of Jiangsu Province, Nanjing 210009, Jiangsu, China

3. Department of Oncology, Zhongda Hospital, School of Medicine, Southeast University, Nanjing 210000, Jiangsu, China

\section{Corresponding Author:}

Feng Jiang

No.42, Baiziting, Xuanwu District, Nanjing 210009, Jiangsu, China

Email address: jiangfengj20@sina.com or fengjiang_nj@njmu.edu.cn

\section{Abstract}

Background: Lung adenocarcinoma (LUAD) is the most common histologic subtype of lung cancer. Studies have found that miR-1293 is related to the survival of LUAD patients. Unfortunately, its role in LUAD remains not fully clarified.

Methods: miR-1293 expression and its association with LUAD patients' clinical characteristics were analyzed in TCGA database. Also, miR-1293 expression was detected in LUAD cell lines. Cell viability, migration, invasion and expression of MMP2 and MMP9 were measured in LUAD cells following transfection with miR-1293 mimic or antagomir. Phosphoglucomutase (PGM) 5 was identified to be negatively related to miR-1293 in LUAD patients in TCGA database, and their association was predicated by Targetscan software. Hence, we further verified the relationship between miR-1293 and PGM5. Additionally, the effect and mechanism of miR-1293 were validated in a xenograft mouse model.

Results: We found miR-1293 expression was elevated, but PGM5 was decreased, in LUAD patients and cells. Higher miR-1293 expression was positively related to LUAD patients' pathologic stage and poor overall survival. miR-1293 mimic significantly promoted, whereas miR-1293 antagomir suppressed the viability, migration, invasion, and expression of MMP2 and MMP9 in LUAD cells. PGM5 was a target of miR-1293. Overexpression of PGM5 abrogated the effects of miR-1293 on the malignant phenotypes of LUAD cells. Administration of miR-1293 antagomir reduced tumor volume and staining of Ki-67 and MMP9, but elevated PGM5 expression in vivo. 
38 Conclusions: miR-1293 promoted the proliferation, migration and invasion of LUAD cells via 39 targeting PGM5, which indicated that miR-1293 might serve as a potential therapeutic target for 40 LUAD patients.

41

\section{Introduction}

Lung cancer is the leading cause of cancer-related death worldwide (Bray et al. 2018). Lung cancer is

divided into two major subtypes, non-small cell lung cancer (NSCLC) and small cell lung cancer (SCLC),

which accounts for $85 \%$ and $15 \%$ of all cases, respectively. NSCLC is composed of three types: mucus and other substances (Denisenko et al. 2018). The 5-year overall survival rate of patients with LUAD is relatively low, with only about $18.1 \%$. This may be attributed, its part, to the complexity of its molecular etiology (Alam et al. 2018; Cancer Genome Atlas Research 2014). Hence, it is of great necessity to have a better understanding of the mechanisms of LUAD tumorigenesis. coding RNA molecules. miRNAs are often aberrantly expressed and act as tumor promotors or suppressors in many different types of cancers including lung cancer (Davalos et al. 2020; Kandettu et al. 2020; Wu et al. 2019). Studies have verified that miRNAs negatively mediate gene expression at the posttranscriptional level via binding to the 3'-untranslated regions (UTRs) of target mRNAs through specific base pairing (Du et al. 2020; Kandettu et al. 2020). A recent study has found that miR-1293 expression is significantly related to the survival of LUAD patients (Wang et al. 2020). However, its effects on the malignant phenotypes of lung cancer especially LUAD are unclear. carcinoma (LUSC) using data from The Cancer Genome Atlas (TCGA) database. The influences of miR- 
67 treatment of miR-1293 mimic or antagomir. Importantly, the specific mechanism involving in tumor-

68 promoting role of miR-1293 was further explored. Additionally, the role and mechanism of miR-1293 in

69 LUAD were further confirmed in a mouse xenograft model.

70

\section{Materials \& Methods}

\section{Analysis of TCGA database and bioinformatics analysis}

73 The expression levels of miR-1293 and phosphoglucomutase (PGM) 5 in LUAD and/or LUSC patients

74 were analyzed in TCGA database using Starbase v3.0 (http://starbase.sysu.edu.cn/) and/or GEPIA2

75 (http://gepia2.cancer-pku.cn/) online software. The correlation between miR-1293/PGM5 and patients'

76 pathologic stage, overall survival, or frequent mutations (eg. KRAS, EGFR, ALK, and PIK3CA) as well

77 as the relationship between miR-1293 and PGM5 were assayed in TCGA database via linkedomics

78 (http://www.linkedomics.org/), Starbase v3.0 or GEPIA2 software. RNA expression profiles in the

79 GSE1 18370 were downloaded from the Gene Expression Omnibus (GEO) database

80 (https://www.ncbi.nlm.nih.gov/geo/), and differentially expressed genes in LUAD tissues were screened by GEO2R with thresholds of $\mid \log 2$ FC (fold-change) $\mid>2$ and $P<0.01$. The target genes of miR-1293 were analyzed by TargetScan (version 7.2).

\section{Cell culture}

84 This study used three LUAD cell lines A549, H1299 and H1975, two LUSC cell lines H226 and H520, 85 and one normal lung cell line BEAS-2B. H520 cells were from the American Type Culture Collection 86 (ATCC; Manassas, VA, USA), and other cell lines were obtained from the Cell Bank of the Chinese 87 Academy of Sciences (Shanghai, China). They were all grown in RPMI-1640 medium, containing 10\% 88 fetal bovine serum (FBS; Gibco, MD, USA) and 1\% penicillin-streptomycin (Gibco) at $37{ }^{\circ} \mathrm{C}$ with $5 \%$ $89 \mathrm{CO}_{2}$.

90 Plasmid construction and cell transfection

91 For the PGM5 overexpression study, PGM5 cDNA amplified from BEAS-2B cells was cloned into the 92 pcDNA3.1 vector to generate pcDNA3.1-PGM5 plasmid. All the transfection processes were carried out 
93 with Lipofectamine 3000 (Invitrogen, Carlsbad, CA, USA). $2 \mu \mathrm{g}$ of constructed vector or pcDNA3.1

94 empty plasmid was transfected into H1299 cells. miR-1293 mimic, miR-1293 antagomir (anti-miR-1293),

95 and their negative controls were synthesized by RiboBio co., LTD (Guangzhou, China). $50 \mathrm{nM}$ of miR-

961293 mimic or $100 \mathrm{nM}$ of miR-1293 antagomir was transfected into H1975 and H1299 cells.

\section{Dual-luciferase assay}

98 The 3'-UTR sequences of PGM5 containing miR-1293 binding sites were amplified from BEAS-2B cells

99 and inserted into the pGL3 reporter vector (Promega, Madison, WI, USA). Also, the mutant seed region

100 of PGM5 was cloned into the pGL3 vector. H1975 cells were plated in a 24-well plate and then

101 transfected with miR-1293 mimic or control at a final concentration of $50 \mathrm{nM}$, and $25 \mathrm{ng}$ of generated

102 PGM5 wild-type (PGM5-WT) or mutant luciferase reporter (PGM5-MUT) using Lipofectamine 3000. 48

$103 \mathrm{~h}$ after transfection, cells were harvested to detect luciferase activity using a Dual-Luciferase Reporter

104 Assay System (Promega).

\section{Cell proliferation analysis}

106 Cell Counting Kit-8 (CCK-8) was used to examine the proliferation of H1975 and H1299 cells. Cells

107 transfected with indicated vectors were seeded into 96 -well plates at a density of $1 \times 10^{3}$ cells/well. After

108 culturing for $0,24,48$ and $72 \mathrm{~h}$, respectively, $10 \mu \mathrm{L}$ of the CCK-8 solution was added to each well. $2 \mathrm{~h}$

109 later, the optical density (OD) value was read by a micro-plate reader (Bio-Tek, Winooski, USA) at 450

$110 \mathrm{~nm}$.

111 Transwell migration and invasion assay

112 The migration and invasion of H1975 and H1299 cells were determined by transwell analysis (Yu et al.

113 2020). For migration assay, the transfected H1975 and H1299 cells were trypsinized and resuspended in

114 RPMI 1640 medium without FBS to adjust the density to $1 \times 10^{5}$ cells $/ \mathrm{mL}$, respectively. In the following,

$115200 \mu \mathrm{L}$ of the cell suspension without FBS was placed into the upper chamber of transwell ( $8 \mu \mathrm{m}$ in pore

116 size, Millipore, Burlington, USA), whereas the lower chamber was filled with $600 \mu \mathrm{L}$ of RPMI 1640

117 medium supplemented with $10 \%$ FBS. After incubating at $37^{\circ} \mathrm{C}$ for $48 \mathrm{~h}$, the upper surface of the

118 membrane was cleaned by cotton swabs, and cells on the lower surface were fixed and stained with $0.1 \%$

Peer) reviewing PDF | (2021:05:60981:1:1:NEW 26 Jul 2021) 
119 crystal violet for $30 \mathrm{~min}$. After drying, stained cells were photographed by a light microscope. For

120 invasion analysis, the upper members of transwell inserts were pre-coated with Matrigel, and other

121 operation processes were consistent with the transwell migration assay.

\section{Animal experiments}

123 Twelve 6-8-week-old BALB/c nude mice were obtained from the Shanghai SLAC Laboratory Animal

124 Co., Ltd (Shanghai, China). All mice were raised in specific-pathogen-free conditions under 12/12 cycle

125 of light at room temperature $\left(25-27^{\circ} \mathrm{C}\right)$ and allowed free access to food and water. To investigate the role 126 of miR-1293 in the tumorigenesis of LUAD, H1975 cells $\left(2 \times 10^{6}\right)$ were subcutaneously injected into the

127 right flank of each mouse. One week later, mice were randomly divided into two groups (n=6 per group):

128 anti-miR-1293 group and anti-NC group. Mice were intratumorally injected with $5 \mathrm{nM}$ miR-1293

129 antagomir or control, respectively, twice a week for 3 weeks. Tumor volumes were measured every week 130 and calculated with the formula $\mathrm{V}\left(\mathrm{mm}^{3}\right)=1 / 2\left(\right.$ length $\times$ width $\left.{ }^{2}\right) .5$ weeks after inoculation, mice were 131 sacrificed by cervical dislocation after inhalational anesthesia with $2 \%$ isoflurane, and the xenografted 132 tumors were removed for analysis. A part of tumor tissues was directly stored at $-80^{\circ} \mathrm{C}$, which was used 133 to detect the expression of miR-1293 and PGM5. The other part of tumor specimens was fixed in 134 formalin and embedded in paraffin to perform immunohistochemical staining for Ki-67 and MMP9. All 135 procedures involving in animals were reviewed and approved by the Affiliated Cancer Hospital of 136 Nanjing Medical University (No. 2020-005).

\section{Real-time PCR}

138 Total RNA was isolated from cells and tissues using TRIzol reagent (Invitrogen) following the 139 manufacturer's instructions. cDNA was synthesized by reverse transcription with the reverse transcription 140 kit (Takara, Dalian, China). The relative levels of PGM5 transcripts were detected by Real-time PCR 141 using the SYBR Green mix (Kakara, Dalian, China). The expression of miR-1293 was determined by

142 TaqMan MicroRNA assay kits (Applied Biosystems, Foster City, CA, USA). The relative fold changes of 143 candidate genes were assayed by the $2^{-\Delta \Delta \mathrm{CT}}$ method, with GAPDH or U6 as an internal control. The 144 primers used were as follows: PGM5, forward, 5'- GATGCTGATGGGGACCGTTA-3' and reverse, 5'- 
145 GCAACGTCCTGAGTCCATCA-3'; GAPDH, forward, 5'-GAAGACGGGCGGAGAGAAAC-3' and 146 reverse, 5'-CCCAATACGACCAAATCCGTTG-3'; miR-1293, forward, 5'-

147 ACACTCCAGCTGGGTGGGTGGTCTGGAGAT-3' and reverse, 5'-TGGTGTCGTGGAGTCG-3'; U6,

148 forward, 5'- CTCGCTTCGGCAGCACA-3' and reverse, 5'-AACGCTTCACGAATTTGCGT-3'.

149 Western blot

150 Cells with different treatments and tumor tissues were lysed with RIPA lysis buffer (Sigma-Aldrich, 151 USA) supplemented with protease inhibitors. Protein concentration was quantified using the BCA Protein 152 Assay Kit (Pierce, Rockford, USA). Samples were denatured by heating in a water bath at $100^{\circ} \mathrm{C}$ for 5 153 min. Cellular proteins were separated using SDS-PAGE by $10 \%$ running gel. After transferring proteins 154 onto the PVDF membrane, the membrane was blocked with 5\% defatted milk for $1 \mathrm{~h}$ at room 155 temperature, incubated with primary antibodies, including PGM5 (1:500; Novus, St. Louis, MO, USA), MMP2 (1:1000; Cell Signaling Technology, Danvers, MA, USA) and MMP9 (1:1000, Cell Signaling Technology) overnight at $4^{\circ} \mathrm{C}$, and probed with horseradish peroxidase (HRP)-conjugated secondary antibody (1:5000). After that, the blots were detected using enhanced chemiluminescent reagents. The relative levels of proteins were analyzed by densitometric analysis using ImageJ software, with GAPDH as an internal control.

\section{Statistical analysis}

All experiments were performed in triplicates and mean \pm standard deviation (SD) was calculated. Data were analyzed by the Graphpad Prism 5 software. Student's $t$ test and one-way analysis of variance (ANOVA) following Bonferroni or Dunnett's post-test analysis were used to perform all statistical analyses. A $P$ value of $<0.05$ is considered statistically significant.

166

167

168

169

170

\section{Results}

\section{miR-1293 expression is elevated in tumor tissues of lung adenocarcinoma patients}

miR-1293 expression was assayed in TCGA database using Starbase online software, and the results indicated that compared with normal samples, miR-1293 expression was upregulated in NSCLC samples, 
171 including adenocarcinoma $(P=0.0065)$ and squamous cell carcinoma $\left(P=2.0 \times 10^{-12}\right)$ (Figure $1 \mathrm{~A}$ and $\left.1 \mathrm{~B}\right)$.

172 Furthermore, miR-1293 expression level was related to the pathologic stage of LUAD patients (Figure

$\left.1731 \mathrm{C}: \mathrm{N}=445 ; P=1.916 \times 10^{-5}\right)$. Higher miR-1293 expression was correlated with poor overall survival of

174 LUAD patients (Figure 1D: $\mathrm{N}=430 ; P=2.621 \times 10^{-6}$ or Figure $1 \mathrm{E}: \mathrm{N}=503 ; P=2.5 \times 10^{-5}$ ). However, we did

175 not find any correlation between miR-1293 expression and the pathologic stage (Figure 1F: N=339;

$176 P=0.7166$ ) or overall survival (Figure $1 \mathrm{G}: \mathrm{N}=325 ; P=0.7199$ ) of LUSC patients. Specially, we further

177 investigated the correlation between miR-1293 expression and the frequent mutations present in LUAD

178 patients and found that miR-1293 expression was positively related to KRAS and PIK3CA mutations, but

179 negatively related to EGFR mutation in LUAD patients, without any correlation with ALK mutation

180 (Figure $1 \mathrm{H}-1 \mathrm{~K}$ ). miR-1293 expression was notably elevated in LUAD cell lines, including A549, H1299

181 and H1975 compared with the normal lung cell line (BEAS-2B). Although miR-1293 expression in

182 LUSC cell line H520 was higher than that in BEAS-2B, we did not find any significant change between

183 H226 and BEAS-2B cells (Figure 1L). Collectively, these results indicated that miR-1293 was increased

184 in LUAD and elevated miR-1293 might serve as a promising prognostic biomarker for LUAD patients.

185

186

187

188

189

190

191

192

193

194

miR-1293 promotes the proliferation, migration and invasion of lung adenocarcinoma cells

We further investigated the role of miR-1293 in LUAD cells. The viabilities of H1975 and H1299 cells

were significantly promoted after miR-1293 mimic transfection (Figure 2A and 2B). The numbers of migrated and invasive cells were increased by miR-1293 mimic (Figure 2C and 2D). Moreover, the mRNA and protein expression levels of MMP2 and MMP9 were elevated by miR-1293 mimic both in H1975 and H1299 cells (Figure 2E-2G). In contrast, miR-1293 antagomir suppressed the viabilities, decreased cell migration and invasion, and reduced the expression of MMP2 and MMP9 in LUAD cells (Figure 2A-2G). To exclude the influence of cell proliferation on cell migration and invasion, LUAD cells in the upper chamber were cultured with RPMI 1640 medium without FBS, which were shown in Supplemental Figure 1. In general, these data indicated that miR-1293 could facilitate the proliferation, migration and invasion of LUAD cells in vitro.

PGM5 as a target of miR-1293 in lung adenocarcinoma

Peer] reviewing PDF | (2021:05:60981:1:1:NEW 26 Jul 2021) 

data, which revealed genes negatively associated with miR-1293 expression in LUAD; the potential target genes of miR-1293 analyzed by TargetScan; and differentially expressed genes in LUAD tissues from the GSE118370. Overlap of the three datasets was displayed using a Venn diagram. We found that 5 genes

201

202

203

204

205

206

207

208

209

210

211

212

213

214

215

216

217

218

219

220

221 were common to all three datasets (Figure 3A). Among these genes, PGM5 was selected, because it has been found to be related to tumor progression in some cancers (Jiao et al. 2019; Sun et al. 2019). As shown in Figure 3B, PGM5 expression showed an inverse correlation with miR-1293 in LUAD patients. We found that PGM5 expression was decreased in tumor tissues of LUAD patients (Figure 3C). Lower PGM5 expression was related to the poor overall survival of LUAD patients (Figure 3D). Furthermore, the mRNA and protein expression levels of PGM5 were reduced in LUAD cell lines compared with BEAS-2B cells (Figure 3E and 3F).

The binding sites between PGM5 mRNA and miR-1293 are shown in Figure 4A. Subsequently, we constructed wild-type and mutated PGM5 dual-luciferase reporter vectors to validate whether PGM5 was a target of miR-1293 in LUAD. The data demonstrated that miR-1293 dramatically decreased the luciferase activities of PGM5 by directly bounding to its 3'UTR, without any effect on the luciferase activities of PGM5-MUT vector (Figure 4B). Additionally, we found that miR-1293 significantly decreased the mRNA and protein levels of PGM5 in H1975 and H1299 cells (Figure 4C-4E). Taken together, these results suggested that PGM5 was a target of miR-1293 in LUAD. miR-1293 functions via regulating PGM5 expression in lung adenocarcinoma cells

To explore whether miR-1293 inhibited LUAD cells' proliferation, migration, and invasion via mediating PGM5, we upregulated PGM5 expression in H1299 cells transfected with or without miR-1293 mimic. Western blot assay showed that pcDNA3.1-PGM5 plasmid significantly enhanced PGM5 expression in H1299 cells (Figure 5A). PGM5 upregulation notably inhibited cell viability and abrogated the promotion of miR-1293 on the viability of H1299 cells (Figure 5B). Using transwell assays, we found that overexpression of PGM5 decreased the numbers of migratory and invasive cells and reversed the effects of miR-1293 on the migration and invasion of H1299 cells (Figure 5C and 5D). Also, PGM5 upregulation 
223 decreased the expression of MMP2 and MMP9 that was increased by miR-1293 mimic (Figure 5E and

224 5F). Therefore, these data further confirmed that miR-1293 functioned via regulating PGM5 expression in

225 LUAD cells.

226 miR-1293 antagomir suppresses LUAD growth and metastasis via negatively modulating PGM5

227 level in vivo

228 The role and mechanism of miR-1293 in LUAD were further evaluated in H1975 xenografted mouse 229 model (Figure 6A). Administration of anti-miR-1293 significantly reduced miR-1293 expression, 230 accompanied by elevated PGM5 expression in tumor tissues (Figure 6B and 6C). Tumor volume and Ki23167 expression were reduced by anti-miR-1293 (Figure 6D-6F). Additionally, MMP9 levels were also 232 decreased in mice treated with anti-miR-1293 (Figure 6F). Taken together, these results indicated that 233 miR-1293 inhibitor could suppress the growth and metastasis of LUAD via negatively modulating PGM5 234 level in vivo.

235

\section{Discussion}

237 Accumulating evidence has demonstrated that a variety of miRNAs are associated with the progression of 238 lung cancer (Liu et al. 2020a; Song et al. 2020). miR-1293 expression was dramatically upregulated in 239 gingivo buccal cancer samples as well as two types of precancer (leukoplakia and lichen planus) tissues

240 (De Sarkar et al. 2014). It was also increased in pancreatic tumor tissues when compared to adjacent non-

241 tumor pancreatic tissues. High expression of miR-1293 showed a reduced overall survival of pancreatic

242 adenocarcinoma patients (Shi et al. 2018). In renal cell carcinoma, miR-1293 was highly expressed, and 243 patients with higher miR-1293 had an unfavorable prognosis. Upregulation of miR-1293 promoted cell 244 viability, migration and invasion in renal cell carcinoma cells (Liu et al. 2020b). However, Takagawa et 245 al. (Takagawa et al. 2020) found that miR-1293 was a tumor-suppressive miRNA and it suppressed colon 246 cancer cell growth both in vitro and in vivo. Currently, its role in lung cancer, especially in LUAD 247 remains poorly understood. 
248 Recent studies have revealed that miR-1293 is an independent prognostic factor in LUAD patients and it 249 is involved in the survival of these patients (Wang et al. 2020; Zheng et al. 2018). Consistent with these 250 results, we found miR-1293 expression was associated with the pathologic stage of LUAD patients, and 251 patients with higher miR-1293 expression showed poor overall survival. Analyzing the expression level 252 of miR-1293 in LUAD patients in TCGA database indicated that miR-1293 expression was upregulated 253 in LUAD samples when compared with normal samples. Interestingly, we found that miR-1293

254 expression was positively related to KRAS and PIK3CA mutations, but negatively related to EGFR 255 mutation in LUAD patients. Similarly, there was an increase of miR-1293 in LUAD cell lines relative to a 256 normal lung cell line. Hence, we hypothesized that miR-1293 might be a tumor promotor in LUAD. In 257 this study, we also found miR-1293 expression was elevated in human LUSC samples. However, its level 258 was not associated with patients' pathologic stage and overall survival. Although miR-1293 expression in 259 LUSC cell line H520 was higher than that in BEAS-2B, we did not find significant change between H226 260 and BEAS-2B cells, this difference may be due to cell lines were established in different ways. Given that 261 miR-1293 expression was not related to the clinical characteristic of LUSC patients, we did not further investigate the role and mechanism of miR-1293 in LUSC.

263 Subsequently, we investigated the effects of miR-1293 on the malignant phenotypes of LUAD cells. In 264 vitro experiments verified that miR-1293 could promote the proliferation, migration and invasion of 265 LUAD cells. On the contrary, miR-1293 antagomir suppressed these characteristics of LUAD cells. 266 Moreover, we found that miR-1293 antagomir reduced tumor growth and Ki-67 staining in a xenograft 267 mouse model. MMP2 and MMP9 are important proteolytic enzymes that degrade collagen type IV, the 268 main component of the basement membrane. They are often hyper-expressed during cancer progression, 269 and related to tumor invasion and metastasis (Shang et al. 2019; Yang et al. 2017; Zhao et al. 2015). In 270 this study, we found that miR-1293 mimic significantly upregulated MMP2 and MMP9 expression, but 271 their levels were reduced by miR-1293 antagomir in LUAD cells. Likewise, MMP9 expression was 272 downregulated by miR-1293 antagomir in tumor tissues of xenografted mice. This further confirmed that 273 miR-1293 could promote the migratory and invasive capabilities of LUAD cells. Taken together, these 
274 data demonstrated that miR-1293 was a tumor promotor that facilitated the proliferation, migration and 275 invasion of LUAD cells.

276 The mechanism of miR-1293 in LUAD was also investigated. Phosphoglucomutases (PGMs) are a 277 family of metabolic enzymes that catalyze the bidirectional interconversion of glucose-1-phosphate and 278 glucose-6-phosphate. It has been demonstrated that PGMs are involved in the proliferation, invasion and 279 metastasis of cancer (Jin et al. 2018; Lee et al. 2010; Ricciardiello et al. 2018). PGM5, which is also 280 known as phosphoglycosidase-related protein (PGM-rp) and Aciculin, is located on human chromosome 2819 (9q21.11) (Edwards et al. 1995). In 1995, Edwards et al. (Edwards et al. 1995) identified that PGM5 is a 282 member of the PGM family. Recent studies found that PGM5 expression is decreased in liver cancer and 283 colorectal cancer, and low PGM5 levels are associated with patients' poor overall survival (Jiao et al. 284 2019; Sun et al. 2019). As well, PGM5 is reported to inhibit the proliferation, invasion and migration 285 abilities of colorectal cancer cells (Sun et al. 2019). By analyzing TCGA database, we found PGM5 286 expression was decreased and negatively involved in miR-1293 in LUAD patients. Lower PGM5 expression was related to the poor overall survival of LUAD patients. Importantly, Targetscan software predicted that PGM5 was a potential target of miR-1293. In this study, we found that PGM5 overexpression could inhibit the proliferation, migration, invasion and expression of MMP2 and MMP9 in LUAD cells. This indicated that PGM5 was a tumor suppressor in LUAD. Using a dual-luciferase assay, we verified that PGM5 was a target of miR-1293 in LUAD cells. miR-1293 could suppress the expression of PGM5 in H1299 and H1975 cells. To further investigate whether miR-1293 functioned via regulating PGM5, we upregulated PGM5 in miR-1293 mimic-transfected H1299 cells and found that PGM5 upregulation reversed the effects of miR-1293 mimic on the proliferation, migration, invasion and expression of MMP2 and MMP9 in this cell line. Moreover, this study found that anti-miR-1293 could elevate PGM5 expression in H1975 xenograft mouse model. In general, these data suggested that miR-

2971293 functioned via targeting PGM5 in LUAD.

\section{Conclusions}


300 Our study found that elevated miR-1293 could promote the proliferation, migration and invasion of

301 LUAD cells. Mechanism exploration verified that miR-1293 played these effects in LUAD via targeting

302 PGM5. This study illustrates comprehensive insights into the roles of miR-1293 and PGM5 in LUAD,

303 which may serve as potential therapeutic targets for LUAD patients.

304

305

306

307

308

309

310

311

312

\section{3}

314

315

316

\section{7}

318

319

320

321

322

323

324

325

326

327

328

329

330

331

332

333

334

335

336

337

338

339

340

341

\section{Acknowledgements}

The study was supported by Youth Foundation of Jiangsu Province (BK20200395). The funders had no role in study design, data collection and analysis, decision to publish, or preparation of the manuscript.

\section{Competing Interests}

The authors declare there are no competing interests.

\section{Data Availability}

The following information was supplied regarding data availability:

The raw measurements are provided in the Supplementary Files.

\section{References}

Alam H, Li N, Dhar SS, Wu SJ, Lv J, Chen K, Flores ER, Baseler L, and Lee MG. 2018. HP1gamma Promotes Lung Adenocarcinoma by Downregulating the TranscriptionRepressive Regulators NCOR2 and ZBTB7A. Cancer Res 78:3834-3848. 10.1158/00085472. CAN-17-3571

Bray F, Ferlay J, Soerjomataram I, Siegel RL, Torre LA, and Jemal A. 2018. Global cancer statistics 2018: GLOBOCAN estimates of incidence and mortality worldwide for 36 cancers in 185 countries. CA Cancer J Clin 68:394-424. 10.3322/caac.21492

Cancer Genome Atlas Research N. 2014. Comprehensive molecular profiling of lung adenocarcinoma. Nature 511:543-550. 10.1038/nature13385

Davalos A, Pinilla L, de Las Hazas ML, Pinto-Hernandez P, Barbe F, Iglesias-Gutierrez E, and de Gonzalo-Calvo D. 2020. Dietary microRNAs and cancer: a new therapeutic approach? Semin Cancer Biol. 10.1016/j.semcancer.2020.10.006

De Sarkar N, Roy R, Mitra JK, Ghose S, Chakraborty A, Paul RR, Mukhopadhyay I, and Roy B. 2014. A quest for miRNA bio-marker: a track back approach from gingivo buccal cancer to two different types of precancers. PLoS One 9:e104839. 10.1371/journal.pone.0104839

Denisenko TV, Budkevich IN, and Zhivotovsky B. 2018. Cell death-based treatment of lung adenocarcinoma. Cell Death Dis 9:117. 10.1038/s41419-017-0063-y

Du X, Wang S, Liu X, He T, Lin X, Wu S, Wang D, Li J, Huang W, and Yang H. 2020. MiR1307-5p targeting TRAF3 upregulates the MAPK/NF-kappaB pathway and promotes lung adenocarcinoma proliferation. Cancer Cell Int 20:502. 10.1186/s12935-020-01595-Z

Edwards YH, Putt W, Fox M, and Ives JH. 1995. A novel human phosphoglucomutase (PGM5) maps to the centromeric region of chromosome 9. Genomics 30:350-353. 10.1006/geno.1995.9866 
342

343

344

345

346

347

348

349

350

351

352

353

354

355

356

357

358

359

360

361

362

363

364

365

366

367

368

369

370

371

372

373

374

375

376

377

378

379

380

381

382

383

384

385

386

387

388

389

390

391
Jiao Y, Li Y, Jiang P, Han W, and Liu Y. 2019. PGM5: a novel diagnostic and prognostic biomarker for liver cancer. PeerJ 7:e7070. 10.7717/peerj.7070

Jin GZ, Zhang Y, Cong WM, Wu X, Wang X, Wu S, Wang S, Zhou W, Yuan S, Gao H, Yu G, and Yang W. 2018. Phosphoglucomutase 1 inhibits hepatocellular carcinoma progression by regulating glucose trafficking. PLoS Biol 16:e2006483. 10.1371/journal.pbio.2006483

Kandettu A, Radhakrishnan R, Chakrabarty S, Sriharikrishnaa S, and Kabekkodu SP. 2020. The emerging role of miRNA clusters in breast cancer progression. Biochim Biophys Acta Rev Cancer 1874:188413. 10.1016/j.bbcan.2020.188413

Lee CH, Jeong SJ, Yun SM, Kim JH, Lee HJ, Ahn KS, Won SH, Kim HS, Lee HJ, Ahn KS, Zhu S, Chen CY, and Kim SH. 2010. Down-regulation of phosphoglucomutase 3 mediates sulforaphane-induced cell death in LNCaP prostate cancer cells. Proteome Sci 8:67. 10.1186/1477-5956-8-67

Liu X, Liu B, Li R, Wang F, Wang N, Zhang M, Bai Y, Wu J, Liu L, Han D, Li Z, Feng B, Zhou G, Wang S, Zeng L, Miao J, Yao Y, Liang B, Huang L, Wang Q, and Wu Y. 2020a. miR146a-5p Plays an Oncogenic Role in NSCLC via Suppression of TRAF6. Front Cell Dev Biol 8:847. 10.3389/fcell.2020.00847

Liu XL, Pan WG, Li KL, Mao YJ, Liu SD, and Zhang RM. 2020b. miR-1293 Suppresses Tumor Malignancy by Targeting Hydrocyanic Oxidase 2: Therapeutic Potential of a miR1293/Hydrocyanic Oxidase 2 Axis in Renal Cell Carcinoma. Cancer Biother Radiopharm 35:377-386. 10.1089/cbr.2019.2957

Read WL, Page NC, Tierney RM, Piccirillo JF, and Govindan R. 2004. The epidemiology of bronchioloalveolar carcinoma over the past two decades: analysis of the SEER database. Lung Cancer 45:137-142. 10.1016/j.lungcan.2004.01.019

Ricciardiello F, Votta G, Palorini R, Raccagni I, Brunelli L, Paiotta A, Tinelli F, D'Orazio G, Valtorta S, De Gioia L, Pastorelli R, Moresco RM, La Ferla B, and Chiaradonna F. 2018. Inhibition of the Hexosamine Biosynthetic Pathway by targeting PGM3 causes breast cancer growth arrest and apoptosis. Cell Death Dis 9:377. 10.1038/s41419-018-0405-4

Shang H, Wang S, Yao J, Guo C, Dong J, and Liao L. 2019. Salidroside inhibits migration and invasion of poorly differentiated thyroid cancer cells. Thorac Cancer 10:1469-1478. 10.1111/1759-7714.13096

Shi XH, Li X, Zhang H, He RZ, Zhao Y, Zhou M, Pan ST, Zhao CL, Feng YC, Wang M, Guo XJ, and Qin RY. 2018. A Five-microRNA Signature for Survival Prognosis in Pancreatic Adenocarcinoma based on TCGA Data. Sci Rep 8:7638. 10.1038/s41598-018-22493-5

Song T, Zhou H, Wei X, Meng Y, and Guo Q. 2020. Downregulation of microRNA-324-3p inhibits lung cancer by blocking the NCAM1-MAPK axis through ALX4. Cancer Gene Ther. 10.1038/s41417-020-00231-2

Sun Y, Long H, Sun L, Sun X, Pang L, Chen J, Yi Q, Liang T, and Shen Y. 2019. PGM5 is a promising biomarker and may predict the prognosis of colorectal cancer patients. Cancer Cell Int 19:253. 10.1186/s12935-019-0967-y

Takagawa Y, Gen Y, Muramatsu T, Tanimoto K, Inoue J, Harada H, and Inazawa J. 2020. miR1293, a Candidate for miRNA-Based Cancer Therapeutics, Simultaneously Targets BRD4 and the DNA Repair Pathway. Mol Ther 28:1494-1505. 10.1016/j.ymthe.2020.04.001

Wang W, Liu B, Duan X, Feng X, Wang T, Wang P, Ding M, Zhang Q, Feng F, Wu Y, Yao W, Wang Q, and Yang Y. 2020. Identification of Three Differentially Expressed miRNAs as Potential Biomarkers for Lung Adenocarcinoma Prognosis. Comb Chem High Throughput Screen 23:148-156. 10.2174/1386207323666200124123103

Wu SG, Chang TH, Liu YN, and Shih JY. 2019. MicroRNA in Lung Cancer Metastasis. Cancers (Basel) 11. 10.3390/cancers 11020265 
392

393

394

395

396

397

398

399

400

401

402

403

404

405

406

407

408

409

410

411

412

413

414

415

416

417

418

419

420

421

422

423

424

425

Yang S, Liu Y, Li MY, Ng CSH, Yang SL, Wang S, Zou C, Dong Y, Du J, Long X, Liu LZ, Wan IYP, Mok T, Underwood MJ, and Chen GG. 2017. FOXP3 promotes tumor growth and metastasis by activating Wnt/beta-catenin signaling pathway and EMT in non-small cell lung cancer. Mol Cancer 16:124. 10.1186/s12943-017-0700-1

Yu ST, Sun BH, Ge JN, Shi JL, Zhu MS, Wei ZG, Li TT, Zhang ZC, Chen WS, and Lei ST. 2020. CRLF1-MYH9 Interaction Regulates Proliferation and Metastasis of Papillary Thyroid Carcinoma Through the ERK/ETV4 Axis. Front Endocrinol (Lausanne) 11:535. 10.3389/fendo.2020.00535

Zhao HJ, Liu T, Mao X, Han SX, Liang RX, Hui LQ, Cao CY, You Y, and Zhang LZ. 2015. Fructus phyllanthi tannin fraction induces apoptosis and inhibits migration and invasion of human lung squamous carcinoma cells in vitro via MAPK/MMP pathways. Acta Pharmacol Sin 36:758-768. 10.1038/aps.2014.130

Zheng R, Mao W, Du Z, Zhang J, Wang M, and Hu M. 2018. Three differential expression profiles of miRNAs as potential biomarkers for lung adenocarcinoma. Biochem Biophys Res Commun 507:377-382. 10.1016/j.bbrc.2018.11.046

\section{Figure legends}

Figure 1 miR-1293 expression in human lung cancer tissues and cell lines. A: miR-1293 expression in human LUAD tissues analyzed by Starbase online software. B: miR-1293 expression in human LUSC samples, which was assayed by Starbase online software. C: The relationship between miR-1293 and the pathologic stage of LUAD patients in TCGA database. The association between miR-1293 and the overall survival of LUAD patients was assayed by linkedomics (D) and starbase (E) software. F: The relationship between miR-1293 and the pathologic stage of LUSC patients. G: The association between miR-1293 and the overall survival of LUSC patients. The correlation between miR-1293 expression and the frequent mutations, including KRAS (H), EGFR (I), ALK (J), and PIK3CA (K) present in LUAD patients. L: miR1293 expression was measured in LUAD cell lines (A549, H1299, and H1975), LUSC cell line (H226 and H520) and normal lung cell line (BEAS-2B). ${ }^{*} P<0.05$ vs the BEAS-2B cells.

Figure 2 miR-1293 facilitates the proliferation, migration and invasion of LUAD cells. CCK-8 assay for the viabilities of H1975 (A) and H1299 (B) cells after transfection with miR-1293 mimic or antagomir (anti-miR-1293) as well as their negative controls. C: The number of migrating cells was elevated by miR-1293 mimic, but decreased by anti-miR-1293 both in H1975 and H1299 cells, which were analyzed by the transwell assay. D: The transwell assay was used to measure the invasion of H1975 and H1299 
426

427

428

429

430

431

432

433

434

435

436

437

438

439

440

441

442

443

444

445

446

447

448

449

450

451

cells. E-G: The protein levels of MMP2 and MMP9 were detected in LUAD cells under different treatments. ${ }^{*} P<0.05$ vs the NC mimic group, ${ }^{\#} P<0.05$ vs the anti-NC group.

Figure 3 PGM5 expression was decreased and negatively associated with miR-1293 in LUAD. A: Venn diagram of specific genes between predicted miR-1293 target genes from Targetscan, TCGA database which showed genes negatively related to miR-1293 in LUAD and differentially expressed genes from the GSE118370. B: The relationship between PGM5 and miR-1293 was analyzed in TCGA database by using linkedomics software. C: PGM5 expression was decreased in LUAD tumor tissues compared with normal samples analyzed by GEPIA2. D: The association between PGM5 and the overall survival of LUAD patients was determined by GEPIA2. E: PGM5 mRNA expression was determined in LUAD cell lines and a normal lung cell line (BEAS-2B). F: Western blot assay for PGM5 protein level in different cell lines. ${ }^{*} P<0.05$ vs the BEAS-2B cells.

Figure 4 PGM5 is a target of miR-1293 in LUAD. A: Targetscan predicted the binding sites between miR-1293 and PGM5. B: H1975 cells were co-transfected with PGM5 wild type (PGM5-WT) or mutant (PGM5-MUT) vectors and miR-1293 mimic or control mimic for $48 \mathrm{~h}$; luciferase activities were detected in these cells following different treatments. C: The mRNA expression of PGM5 was analyzed in H1975 and H1299 cells after transfection with miR-1293 mimic or its control. (D) and (E) PGM protein levels were examined by western blot. ${ }^{*} P<0.05$ vs the NC mimic group.

Figure 5 miR-1293 functions via regulating PGM5 expression in LUAD cells. A: H1299 cells transfected with pcDNA3.1-PGM5 plasmid revealed higher PGM5 expression than that with the pcDNA3.1 vector.

B: Cell viability was determined in H1299 cells under different treatments. (C) and (D) Transwell assays were used to analyze the migration and invasion of H1299 cells. (E) and (F) The protein levels of MMP2 and MMP9 were measured in $\mathrm{H} 1299$ cells. ${ }^{*} P<0.05$ vs the pcDNA3. 1 group, ${ }^{\#} P<0.05$ vs the NC mimic group, ${ }^{\&} P<0.05$ vs the miR-1293 mimic group. 
453 Figure 6 miR-1293 antagomir suppresses LUAD growth and metastasis via negatively modulating PGM5

454 level in vivo. A: A timeline for the H1975 xenograft experiment. B: miR-1293 expression in tumor

455 tissues. C: PGM5 protein level was detected in mouse tumor samples by western blot. D: Mice were

456 sacrificed at 5 weeks after inoculation of H1975 cells, and tumor images were photographed. E: Tumor

457 volumes were measured every week after inoculation. F: Immunohistochemical staining for Ki-67 (upper)

458 and MMP9 (lower) in mouse tumor sections.

459

460

461 Supplemental Figures

462 Figure 1 Cell proliferation in serum-free medium. H1975 and H1299 cells transfected with miR-

4631293 mimic or antagomir (anti-miR-1293) were seeded into 96-well plates and cultured in

464 serum-free RPMI 1640 medium for 48 h, cell viability was analyzed by CCK-8 assay.

465 


\section{Figure 1}

miR-1293 expression in human lung cancer tissues and cell lines

A: miR-1293 expression in human LUAD tissues analyzed by Starbase online software. B: miR-1293 expression in human LUSC samples, which was assayed by Starbase online software. C: The relationship between miR-1293 and the pathologic stage of LUAD patients in TCGA database. The association between miR-1293 and the overall survival of LUAD patients was assayed by linkedomics (D) and starbase (E) software. F: The relationship between miR-1293 and the pathologic stage of LUSC patients. G: The association between miR-1293 and the overall survival of LUSC patients. The correlation between miR-1293 expression and the frequent mutations, including KRAS (H), EGFR (I), ALK (J), and PIK3CA (K) present in LUAD patients. L: miR-1293 expression was measured in LUAD cell lines (A549, H1299, and H1975), LUSC cell line (H226 and H520) and normal lung cell line (BEAS-2B). ${ }^{*} P<0.05$ vs the BEAS-2B cells. 
A

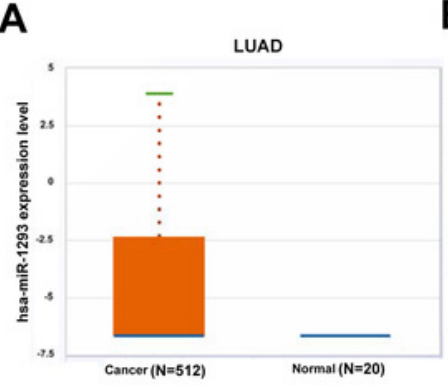

B

E
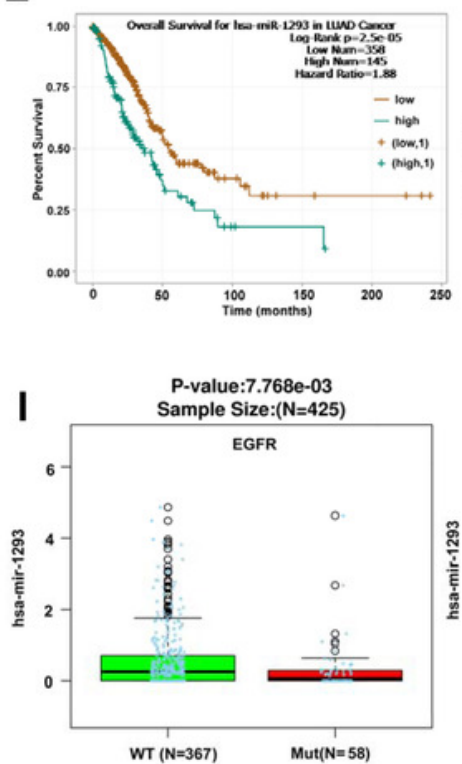
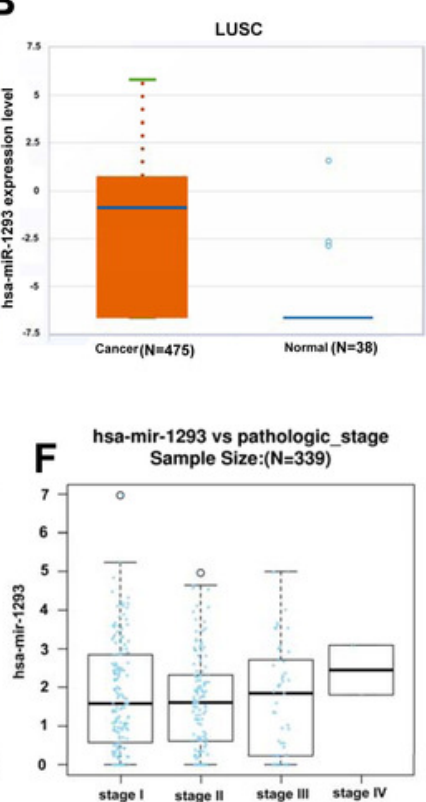

C hsa-mir-1293 vs pathologic_stage

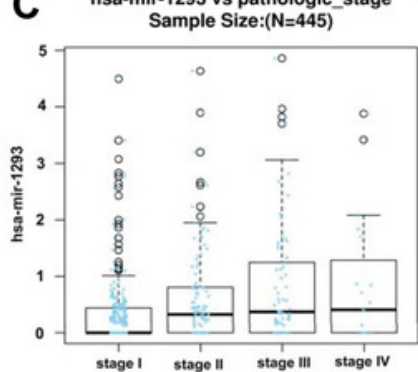

stage I stage II stage III stage IV G $\begin{gathered}\text { hsa-mir-1293 vs overall_survival } \\ (0<=\text { Median })(1>\text { Median }) \text { Median }=1.6012\end{gathered}$
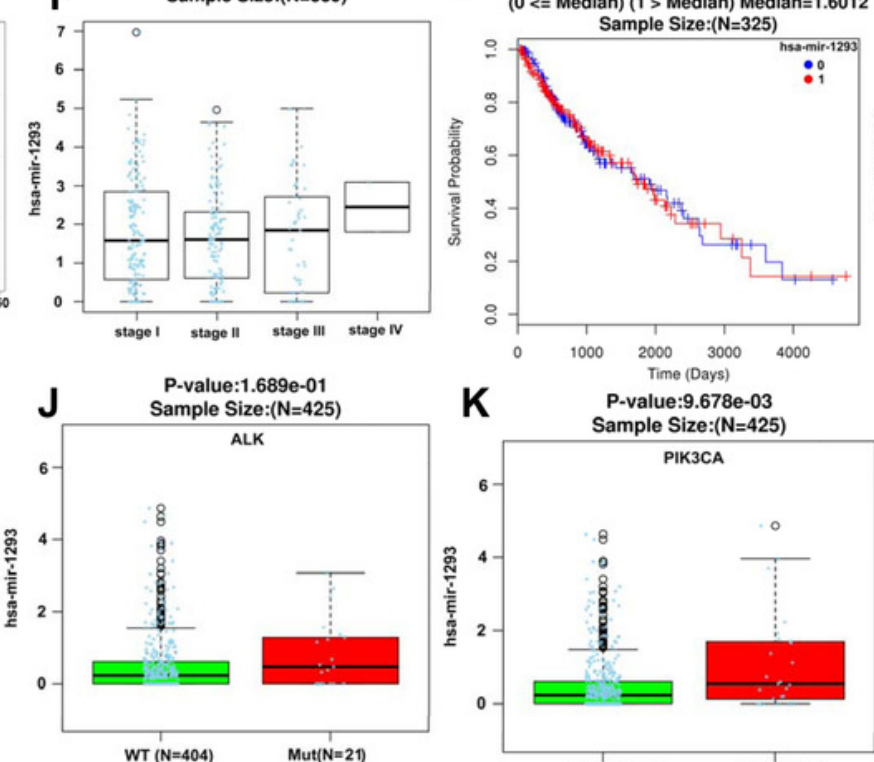

D
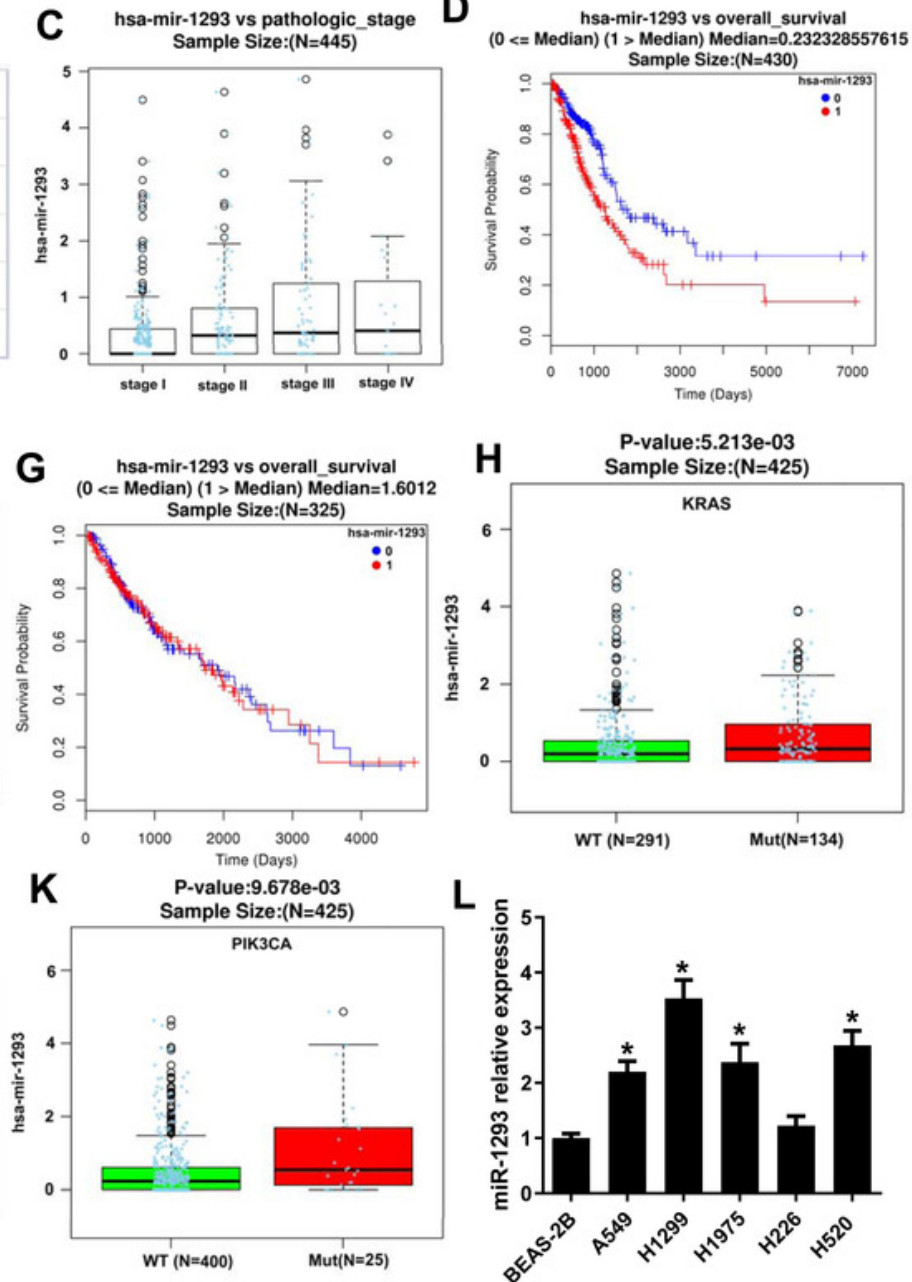

L

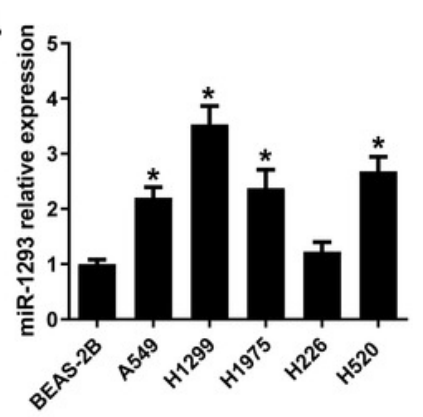




\section{Figure 2}

miR-1293 facilitates the proliferation, migration and invasion of LUAD cells.

CCK-8 assay for the viabilities of H1975 (A) and H1299 (B) cells after transfection with miR-1293 mimic or antagomir (anti-miR-1293) as well as their negative controls. C: The number of migrating cells was elevated by miR-1293 mimic, but decreased by anti-miR-1293 both in $\mathrm{H} 1975$ and H1299 cells, which were analyzed by the transwell assay. D: The transwell assay was used to measure the invasion of H1975 and H1299 cells. E-G: The protein levels of MMP2 and MMP9 were detected in LUAD cells under different treatments. ${ }^{*} P<0.05$ vs the NC mimic group, ${ }^{*} P<0.05$ vs the anti-NC group.
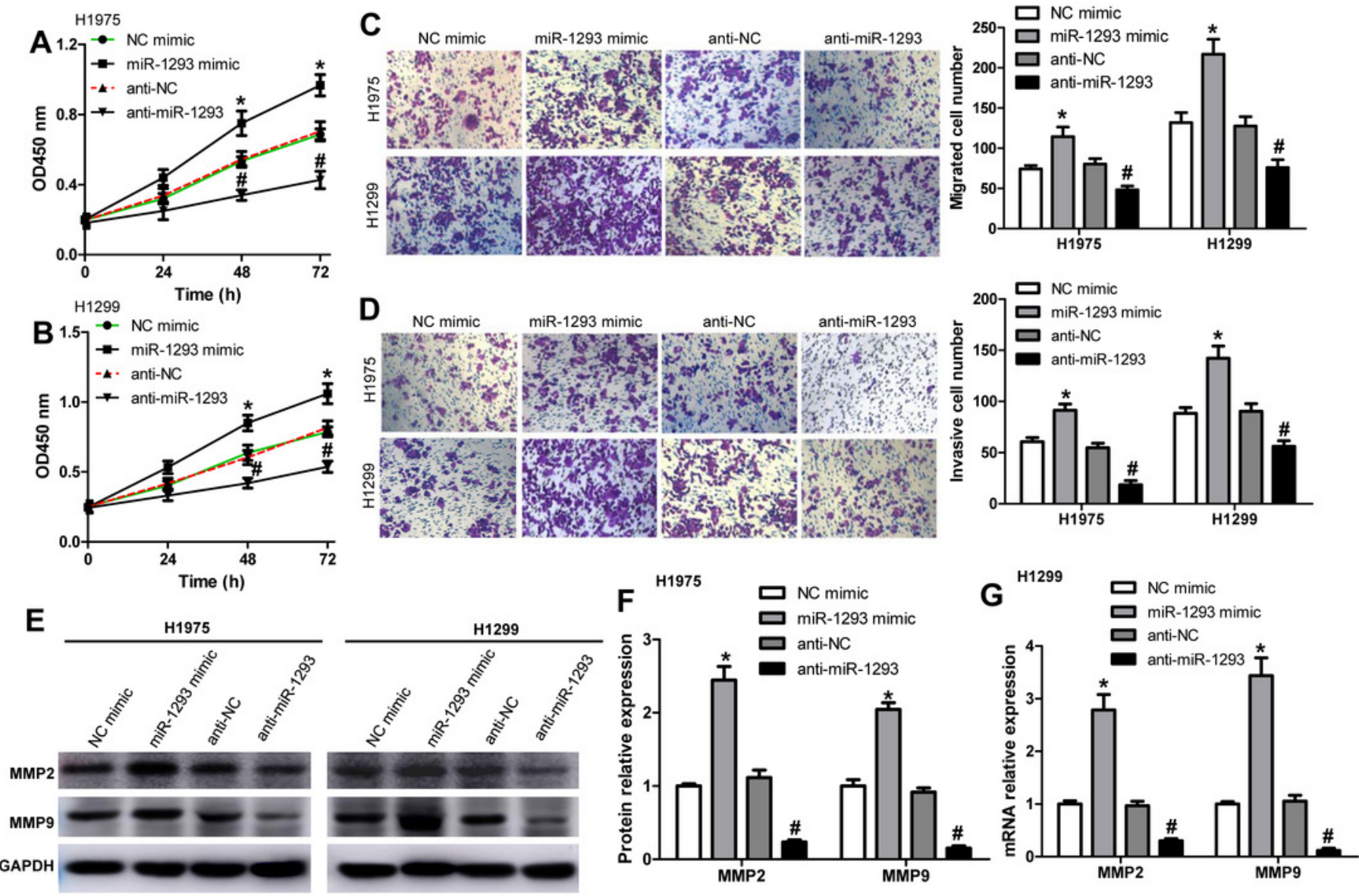


\section{Figure 3}

PGM5 expression was decreased and negatively associated with miR-1293 in LUAD

A: Venn diagram of specific genes between predicted miR-1293 target genes from

Targetscan, TCGA database which showed genes negatively related to miR-1293 in LUAD and differentially expressed genes from the GSE118370. B: The relationship between PGM5 and miR-1293 was analyzed in TCGA database by using linkedomics software. C: PGM5 expression was decreased in LUAD tumor tissues compared with normal samples analyzed by GEPIA2. D: The association between PGM5 and the overall survival of LUAD patients was determined by GEPIA2. E: PGM5 mRNA expression was determined in LUAD cell lines and a normal lung cell line (BEAS-2B). F: Western blot assay for PGM5 protein level in different cell lines. ${ }^{*} P<0.05$ vs the BEAS-2B cells. 
A

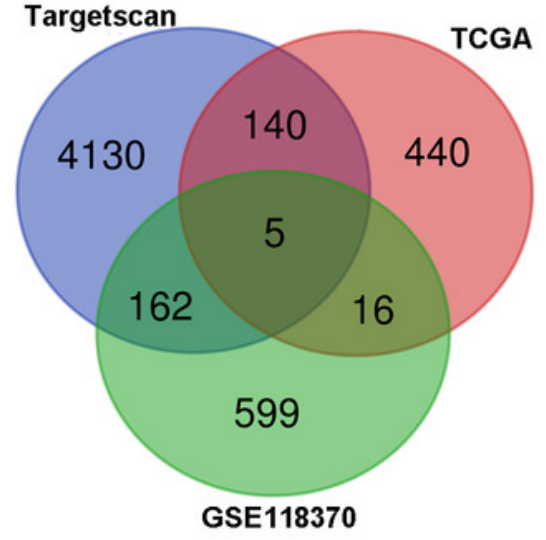

D

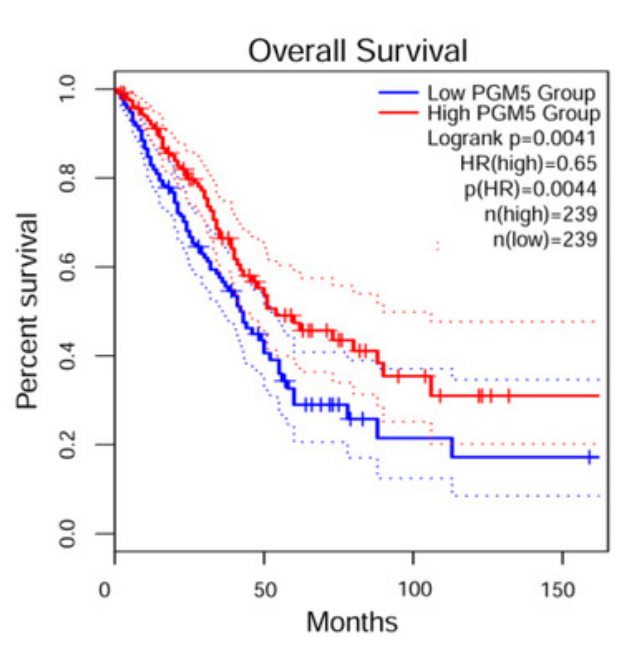

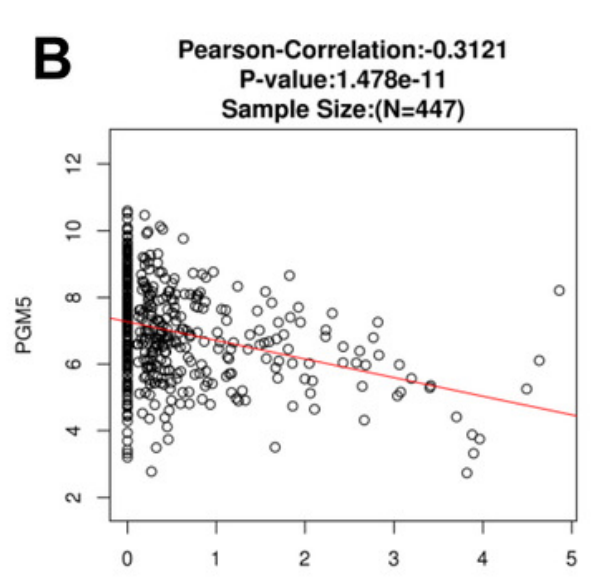

hsa-mir-1293

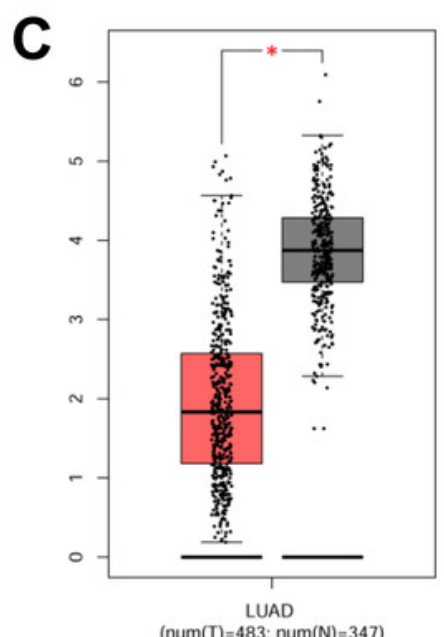

E

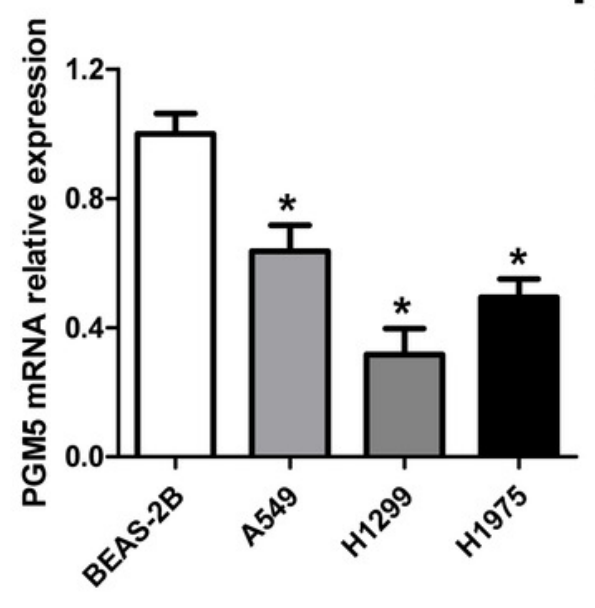

\section{$F_{\text {PGM5 }}$}

GAPDH

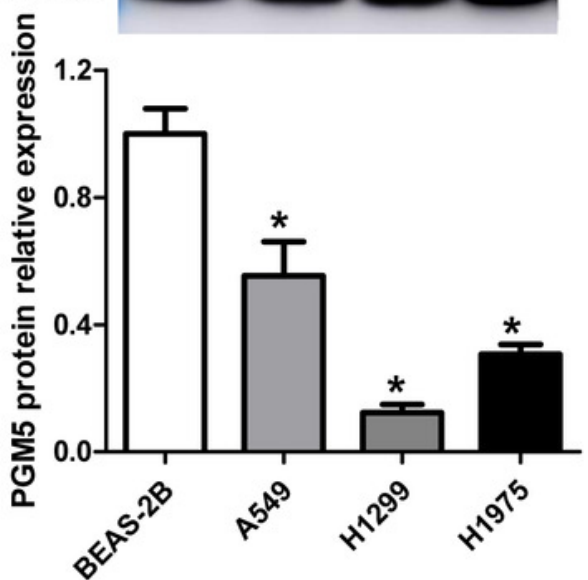




\section{Figure 4}

PGM5 is a target of miR-1293 in LUAD

A: Targetscan predicted the binding sites between miR-1293 and PGM5. B: H1975 cells were co-transfected with PGM5 wild type (PGM5-WT) or mutant (PGM5-MUT) vectors and miR-1293 mimic or control mimic for $48 \mathrm{~h}$; luciferase activities were detected in these cells following different treatments. C: The mRNA expression of PGM5 was analyzed in H1975 and H1299 cells after transfection with miR-1293 mimic or its control. (D) and (E) PGM protein levels were examined by western blot. ${ }^{*} P<0.05$ vs the NC mimic group. 
A miR-1293 3'-CGUGUUUAGAGGUCUGGUGGGU-5'

$$
\text { IIII IIIIIII }
$$

PGM5-WT 5'-CCCACCUCACUCCUG--CCACCCAC-3'

PGM5-MUT 5'-CCCACCUCAGUGCUG--GCUCGCUC-3'
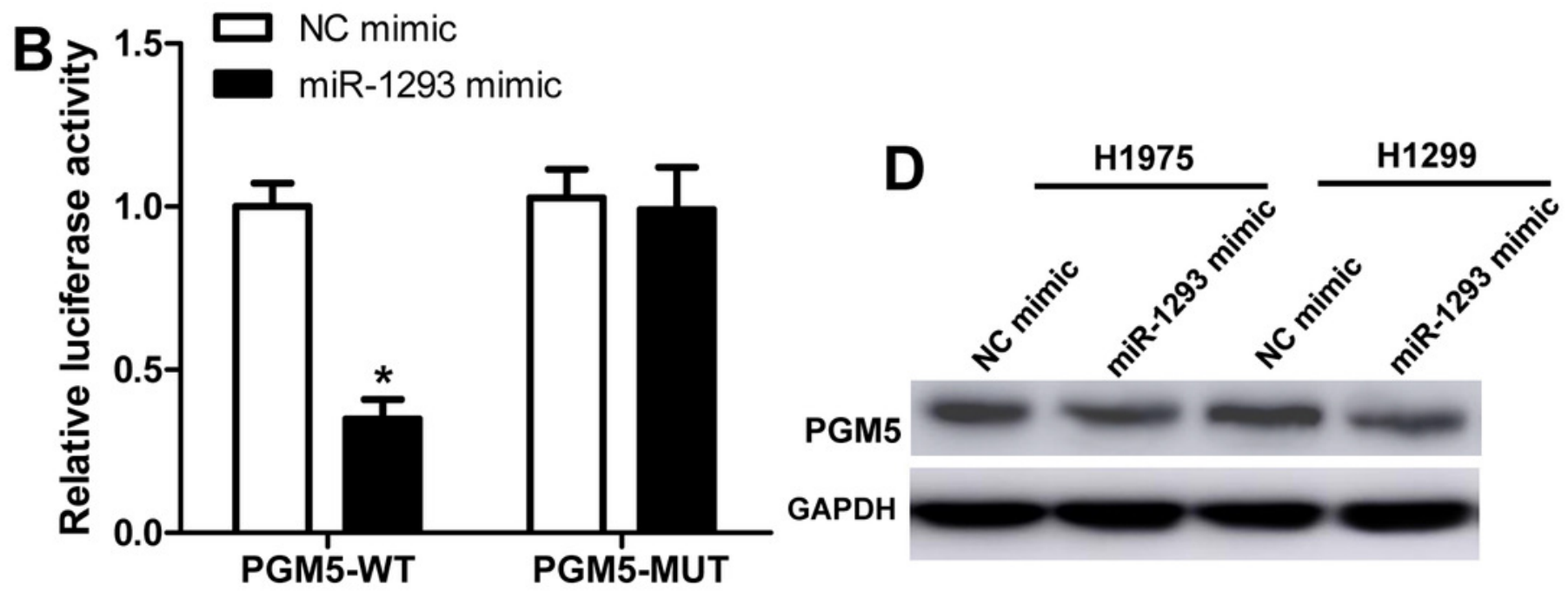

C
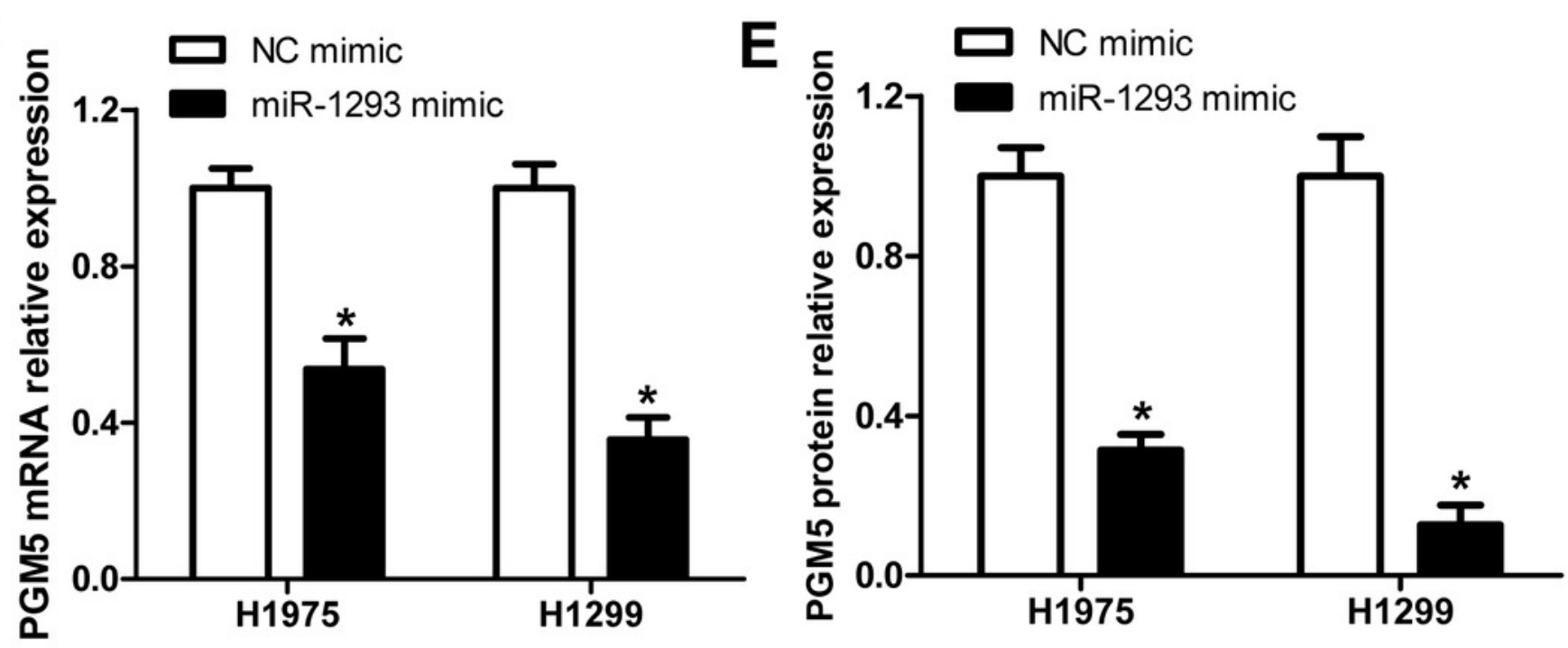


\section{Figure 5}

miR-1293 functions via regulating PGM5 expression in LUAD cells.

A: H1299 cells transfected with pcDNA3.1-PGM5 plasmid revealed higher PGM5 expression than that with the pcDNA3.1 vector. B: Cell viability was determined in H1299 cells under different treatments. (C) and (D) Transwell assays were used to analyze the migration and invasion of H1299 cells. (E) and (F) The protein levels of MMP2 and MMP9 were measured in H1299 cells. ${ }^{*} P<0.05$ vs the pcDNA3.1 group, ${ }^{\#} P<0.05$ vs the NC mimic group, ${ }^{\circledR} P<0.05$ vs the miR-1293 mimic group.

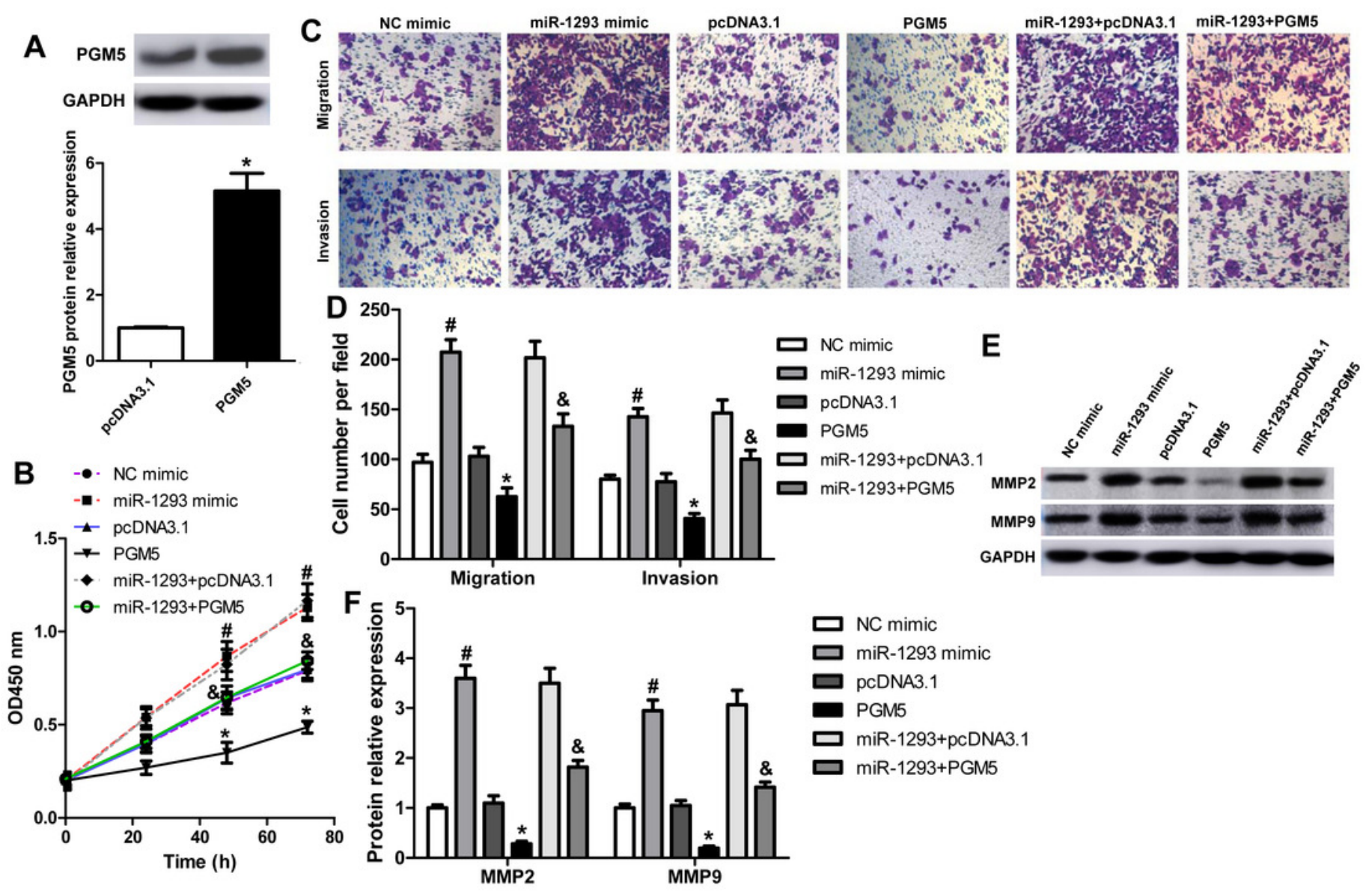




\section{Figure 6}

miR-1293 antagomir suppresses LUAD growth and metastasis via negatively modulating PGM5 level in vivo.

A: A timeline for the H1975 xenograft experiment. B: miR-1293 expression in tumor tissues. C: PGM5 protein level was detected in mouse tumor samples by western blot. D: Mice were sacrificed at 5 weeks after inoculation of H1975 cells, and tumor images were photographed. E: Tumor volumes were measured every week after inoculation. F: Immunohistochemical staining for Ki-67 (upper) and MMP9 (lower) in mouse tumor sections. 


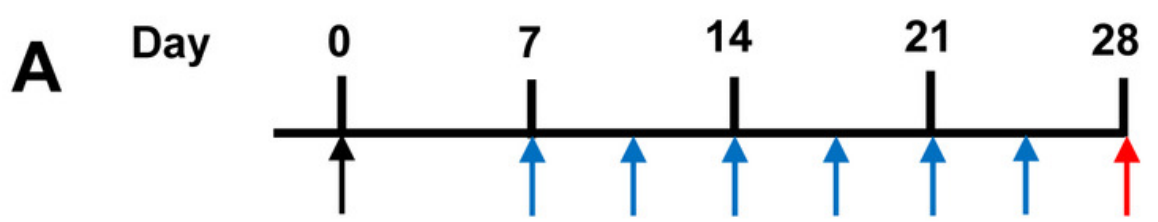

Implant $2 \times 10^{6} \quad$ Intratumorally inject sacrifice H1975 cells in $5 \mathrm{nM}$ miR-1293

B mouse right flank antagomir or control

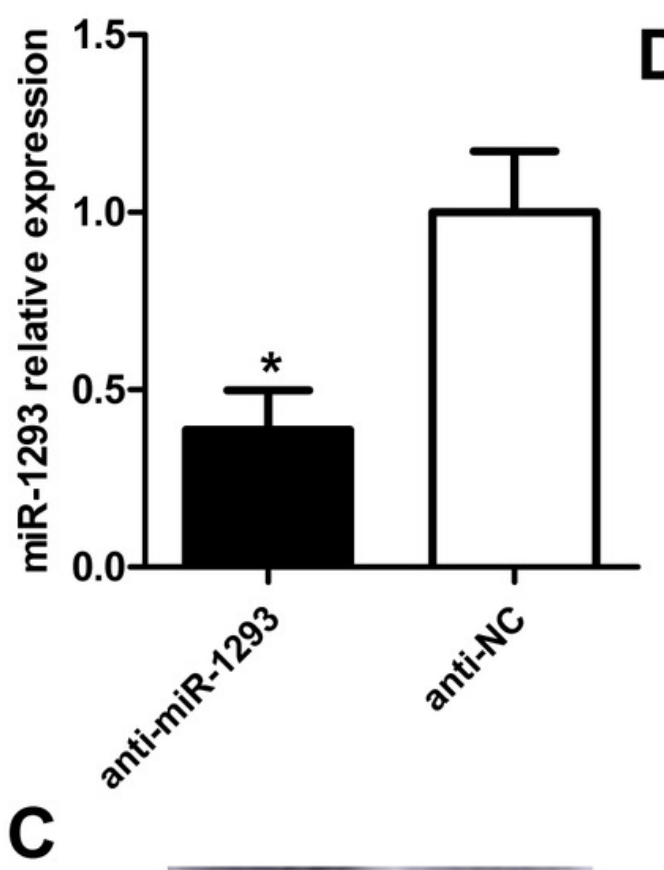

D
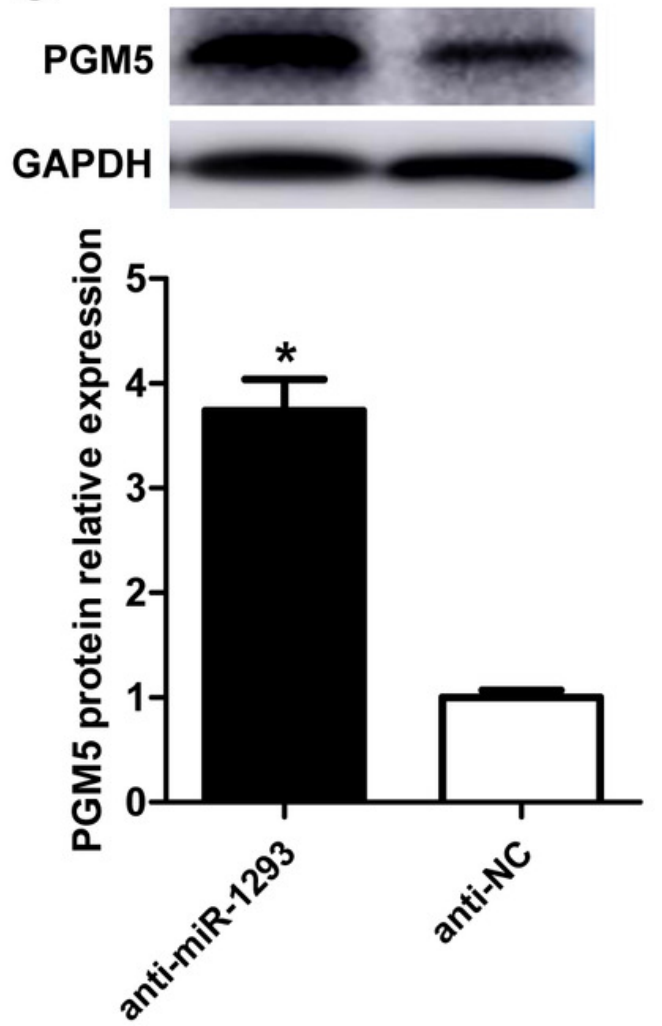

anti-miR-1293

anti-NC

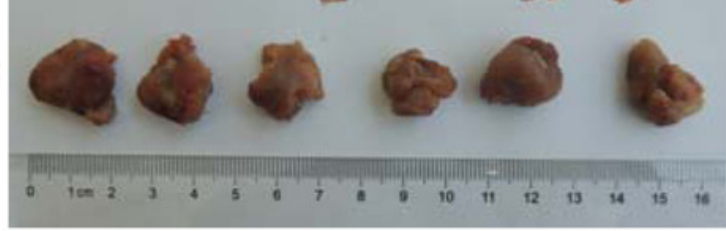

E
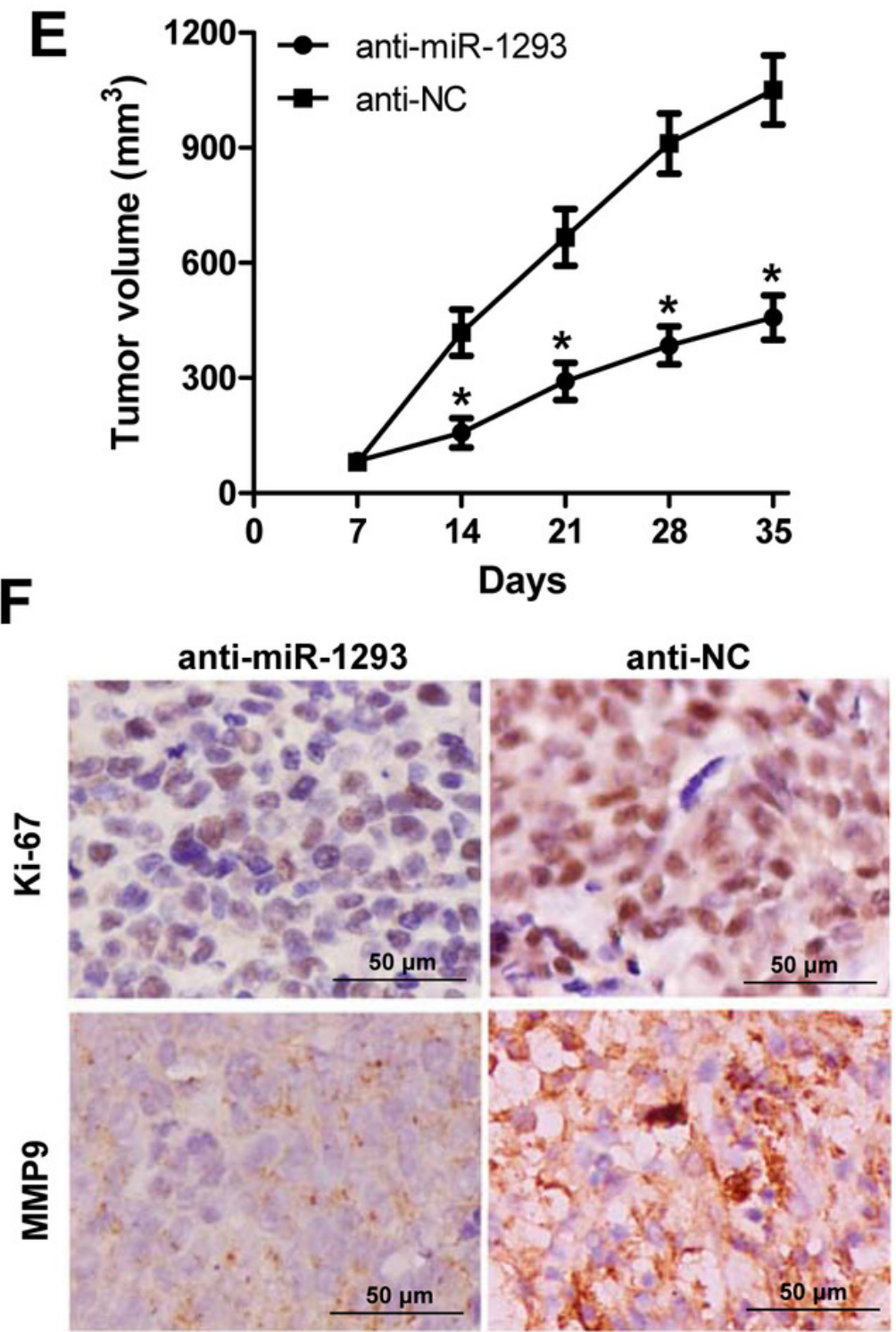